Annuaire suisse de politique de développement

$19 \mid 2000$

Pour une politique plus cohérente envers les pays en développement

\title{
La cohérence des politiques dans les relations Nord-Sud
}

Habib Ouane

\section{CpenEdition}

\section{Journals}

Édition électronique

URL : http://journals.openedition.org/aspd/841

DOI : $10.4000 /$ aspd. 841

ISSN : 1663-9669

Éditeur

Institut de hautes études internationales et du développement

Édition imprimée

Date de publication : 1 avril 2000

Pagination : 103-111

ISSN : 1660-5934

Référence électronique

Habib Ouane, "La cohérence des politiques dans les relations Nord-Sud ", Annuaire suisse de politique de développement [En ligne], 19 | 2000, mis en ligne le 18 août 2012, consulté le 08 septembre 2020. URL : http://journals.openedition.org/aspd/841 ; DOI : https://doi.org/10.4000/aspd.841 


\title{
LA COHÉRENCE DES POLITIOUES DANS LES RELATIONS NORD-SUD
}

\author{
HABIB OUANE*
}

\begin{abstract}
คTTE FIN DE SIÈCLE est émaillée d'une série de paradoxes qui ont eu pour effet de mettre en lumière la pertinence croissante de la cohérence des politiques de coopération internationale au développement. En effet, la prise de conscience de l'interdépendance grandissante entre le Nord et le Sud, l'émergence de problèmes globaux ou transfrontaliers - tels que l'environnement, les droits de l'homme, la finance globalisée, etc. - coexistent avec un amoindrissement graduel des approches globales et multilatérales pour promouvoir des solutions concertées dans un cadre démocratique comme les Nations Unies. En outre, les relations économiques internationales, voire les relations internationales dans leur ensemble sont de plus en plus marquées par le phénomène du pouvoir, ce qui semble contrevenir aux objectifs d'équité et d'équilibre supposés sous-tendre les rapports au sein de la communauté internationale. L'évolution récente de la coopération internationale au développement incite à considérer que la cohérence éthique devrait constituer le fondement préalable et indispensable à toute tentative pour renforcer la cohérence des politiques dans ce domaine. La fin de la guerre froide, la convergence des modes de gestion économique et des systèmes politiques ainsi que l'importance croissante de la société civile dans les relations internationales devraient consolider la cohérence des politiques de coopération au développement.
\end{abstract}

Les objectifs définis en matière de coopération au développement devraient être cohérents et en phase avec ceux poursuivis dans d'autres sphères de la politique de coopération, notamment le commerce, l'environnement, la sécurité et les domaines sociaux. Il est communément admis que l'incohérence des politiques de coopération résulte des conflits d'intérêts et de valeurs d'une part, et de la compartimentation des politiques sectorielles et des structures administratives publiques chargées de leur exécution d'autre part. Il y a lieu de rappeler que dans le domaine de la coopération au développement la problématique de la cohérence des politiques a en principe pour finalité le développement des pays bénéficiaires, fondé sur des intérêts mutuellement avantageux.

\section{LA COHÉRENCE DES POLITIQUES ET LE DÉVELOPPEMENT}

La cohérence des politiques de coopération sera donc analysée dans la perspective des pays en voie de développement (PVD). Pour ce faire, la problématique consistera à déterminer quelles sont les principales questions de cohérence des politiques dans les rapports Nord-Sud en matière de commerce et de développement. Pourquoi se cantonner au commerce notamment? Parce qu'il représente 
le vecteur principal de la mondialisation et a de ce fait la plus grande incidence sur l'orientation des rapports Nord-Sud du point de vue de la cohérence des politiques.

La quête d'une cohérence des politiques ne devrait en principe pas conduire à imposer un modèle de développement aux PVD. Le fait que plusieurs PVD, ayant subi une crise financière et économique, aient été auparavant considérés comme modèles de développement est une manifestation d'une double crise: une crise du développement, mais aussi une crise de la pensée en matière de développement. En fait, les certitudes relatives à la compréhension des exigences d'une stratégie de développement réussie ont été sérieusement ébranlées. En outre, l'idée suivant laquelle le processus de libéralisation et de globalisation représente une marée qui va inévitablement faire progresser tous les navires - petits et grands - n'est plus crédible. Pourquoi ? L'une des raisons est due au fait que l'on a sous-estimé l'importance d'un facteur fondamental: la globalisation et la libéralisation ont particulièrement accentué le degré selon lequel le succès économique des nations, des entreprises, voire des individus dépend d'une acquisition rapide des compétences, de la création et de l'utilisation effective de la technologie et de l'information, ainsi que de leur exploitation à travers les marchés. Cet état de choses a aggravé le fossé Nord-Sud, notamment sur le plan des options disponibles pour les pays développés, riches en compétences, en vue d'accélérer leur croissance et celles - plutôt limitées - dont disposent les pays pauvres, en particulier ceux d'Afrique ou les pays les moins avancés (PMA). En fait, la plupart des PVD n'ont pas réussi à engranger les dividendes de l'intégration dans l'économie mondialisée.

Pourquoi de telles considérations dans une réflexion sur la cohérence des politiques? Parce que l'intégration des PVD dans l'économie mondialisée est, en fait, l'un des défis majeurs en matière de cohérence des politiques. La cohérence des politiques n'est pas un concept neutre, abstrait. Il s'agit d'un concept normatif dont la finalité première doit être la promotion du développement. Nous devons réexaminer les processus, mécanismes et politiques qui sous-tendent le fonctionnement de l'économie mondiale et en particulier ceux qui relient les PVD aux forces de la globalisation. Ce réexamen - dont la cohérence des politiques est l'un des piliers - devrait aborder sans détour la question de savoir comment rendre ces processus plus équitables et plus favorables au développement.

Dans cette perspective, quelles sont les principales questions de cohérence des

* politiques en matière de commerce et de développement dans les relations Nord-Sud, sur les plans tant multilatéral que bilatéral?

Une façon de répondre à cette question consisterait à examiner le degré d'impact positif exercé par les systèmes internationaux sur le développement. Les systèmes internationaux affectant le développement comprennent un ensemble de règles, pratiques et attitudes définies ou promues par les organisations économiques multilatérales et les agences de coopération régionales et bilatérales. Ces systèmes déterminent ce qui représente le cadre ou la norme de politique nationale - pour la plupart des PVD - acceptable sur le plan international. Au centre de ces systèmes figure un modèle de développement destiné à guider la réforme des politiques nationales dans les PVD. Ce que l'on a coutume de dénommer le «Consensus de Washington» participe de cette démarche. Autour de ce modèle central gravitent des sous-systèmes qui gouvernent le commerce, 
les flux financiers et la coopération - bilatérale et multilatérale - au développement, sous-systèmes fortement liés au modèle central. En guise d'illustration, ce modèle fondé sur l'intégration des PVD dans le système mondialisé vient de subir des revers suite à la crise en Asie et dans d'autres pays émergents. Si l'intégration rapide et totale dans l'économie mondiale et dans le système financier et commercial global représente la meilleure stratégie de développement, si le développement est un processus censé réduire la vulnérabilité des économies aux chocs externes, comment peut-on expliquer que les PVD les plus affectés par la dernière crise systémique étaient justement les plus développés du monde en développement et les plus intégrés à l'échelle internationale ?'

La réponse à cette question ne consiste pas à nier l'utilité de l'intégration dans le système économique, financier et commercial global. Plutôt que le degré, l'intensité, la rapidité ou l'ampleur de l'intégration, ce qui importe, c'est sa qualité, sa durabilité et sa séquence.

En fait, le modèle de développement fondé sur le «Consensus de Washington» n'a pas suffisamment pris en charge trois activités clés: s'assurer que les marchés fonctionnent correctement; promouvoir une répartition équitable des coûts et des avantages de la croissance; et, enfin, sécréter un environnement externe porteur et favorable au développement. Du point de vue de la cohérence des politiques, on peut illustrer cette assertion à travers les constats suivants :

• les pressions destinées à libéraliser intégralement les marchés financiers dans les PVD n'ont pas assez tenu compte du comportement de la finance globalisée; cela peut expliquer pourquoi la crise asiatique a eu un fort impact sur des pays très affectés comme la Thaïlande et 1'Indonésie et un impact moindre sur des pays peu affectés comme l'Inde et la Chine. Ces derniers n'ont pas libéralisé leurs marchés financiers et ont peut-être été, de ce fait, moins vulnérables aux effets de contagion de la crise en Asie;

- la libéralisation du commerce dans les PVD n'a pas été accompagnée par des améliorations significatives en suffisance - pour les secteurs où ils sont compétitifs - dans l'accès aux marchés du Nord. Il s'agit là d'un déficit de mise en œuvre des résultats du Cycle d'Uruguay, qui semble de ce point de vue avoir engrangé une libéralisation asymétrique;

- les exigences en matière d'équité n'ont pas été confirmées par l'augmentation des flux d'aide publique aux PVD les plus pauvres notamment.

Pourquoi ces trois illustrations? Parce qu'elles ont comme implication directe que les systèmes internationaux qui sous-tendent le modèle de développement dominant n'ont pas été suffisamment favorables aux PVD et ont parfois produit des effets pervers. Cette question figure à l'épicentre de la problématique de la cohérence des politiques dans le domaine de la coopération au développement, mais aussi dans ceux du commerce et du développement.

1. Il s'agit notamment des «tigres du Sud-Est asiatique» comme la Thaillande, la Malaisie, l'Indonésie, la Corée du Sud - qui avait rejoint le club des pays de l'OCDE; la crise a ensuite frappé la Russie et les PVD les plus avancés d'Amérique du Sud, notamment le Brésil, l'Argentine et le Chili. 


\section{LA COHÉRENCE DES POLITIQUES ET LE COMMERCE}

Le système commercial fondé sur des règles sous-jacentes à l'OMC est supposé atténuer le rôle des pouvoirs économique et politique dans les relations commerciales entre les éléments constitutifs du système que sont les Etats. Les règles elles-mêmes, les thèmes choisis pour les négociations et les modalités asymétriques de libéralisation qui ont émergé du fonctionnement du système commercial multilatéral continuent de refléter le pouvoir relatif des membres de l'OMC. A titre d'illustration, l'accord sur les subventions stipule un haut degré de flexibilité pour les gouvernements concernant les subventions en matière de recherche et de développement (R\&D) et de technologie. Cela permet aux pays qui en ont les moyens de renforcer, à travers les subventions, leur compétitivité internationale pour les exportations qui dépendent de l'avantage technologique. Les résultats de ces percées technologiques étant alors protégés par l'intermédiaire de l'Accord sur les aspects des droits de propriété intellectuelle liés au commerce (ADPIC), ces percées technologiques fournissent une base pour la pénétration du marché à travers le commerce ou l'investissement. Le système commercial multilatéral a tendance à «verrouiller» cet avantage compétitif des pays industrialisés en s'assurant que les marchés demeurent ouverts aux produits découlant de ces percées technologiques. La célérité avec laquelle l'Accord de l'OMC sur les nouvelles technologies a été conclu est une nouvelle illustration de cette tendance.

Les produits d'intérêt à l'exportation pour les PVD sont moins bien traités par le système commercial multilatéral: les matières premières (aliments et fibres) et leurs dérivés transformés - y compris les produits tropicaux - continuent de se heurter à des barrières commerciales élevées dans les pays industriels: les textiles et l'habillement sont sujets à des droits de douane élevés; le report jusqu'à 2005 du démantèlement des restrictions quantitatives auxquelles sont sujettes les exportations de textiles et d'habillement représente une charge inhibant le potentiel d'exportation des PVD.

En fait, depuis le milieu de la décennie 1980, les PVD ont entrepris une libéralisation commerciale rapide et soutenue. Cet état de choses a découlé non seulement des négociations commerciales multilatérales, mais aussi des conditionnalités inhérentes aux programmes d'ajustement structurel et de stabilisation. Le résultat prévisible en a été l'asymétrie croissante entre le Nord et le Sud dans le rythme de la libéralisation, entrainant des conséquences très claires: les exportations d'Amérique latine vers l'Europe ont augmenté de seulement $29 \%$ pendant les années 1990, tandis que celles de l'Europe vers cette région en développement ont augmenté de $164 \%$. Cette disparité est imputable à plusieurs facteurs, parmi lesquels les barrières agricoles européennes figurent en bonne place, d'autant plus que les exportations latino-américaines vers des marchés non européens ont augmenté pendant la même période de $120 \%$. Il résulte de cette asymétrie dans la libéralisation que pour la plupart des PVD la moyenne des déficits commerciaux dans les années 1990 a été supérieure de près de $3 \%$ à celle des années 1970 tandis que le taux de croissance annuelle moyen a été inférieur de $2 \%$, un constat d'autant plus paradoxal que ces PVD ont mené des politiques de réformes macro-économiques et d'ajustement structurel pendant près de deux décennies. 
Du point de vue des PVD, l'absence de cohérence des politiques dans les caractéristiques suivantes du système commercial a contribué à inhiber le développement de leur potentiel à l'exportation :

- l'autorisation de subventions dans les domaines essentiels à la compétitivité de l'exportation des pays industriels (R\&D et crédits à l'exportation), alors que les subventions utilisées par les PVD ont subi des restrictions;

- la libéralisation très embryonnaire du commerce des produits agricoles et l'application très contraignante des dispositions sanitaires et de santé publique qui affectent surtout les exportations des PVD. Il est par exemple notoire que les PVD rencontrent toujours plus de difficultés pour satisfaire aux conditions techniques requises dans les pays industriels importateurs; il en va de même pour les certificats de conformité émis par les institutions nationales des PVD qui ne sont pas reconnus par les pays du Nord;

- la forte protection de l'industrie alimentaire dans les pays du Nord, ce qui a pour effet de handicaper la diversification des activités dans les PVD, notamment pour les produits en croissance rapide comme les fruits et légumes. Il est frappant de constater que les exportations des 48 pays les moins avancés ne représentent que $0,25 \%$ du commerce mondial, ce qui peut difficilement constituer une menace pour les économies les plus puissantes du monde. A titre d'illustration, et selon le directeur général de l'OMC: «C'est triste à dire: les droits de douane moyens appliqués par les pays riches aux produits des pays les plus pauvres sont en fait plus lourds que ceux qu'ils perçoivent sur les produits d'autres pays riches. Aux EtatsUnis et au Canada, par exemple, ces droits de douane sont même environ deux fois plus élevés [...] il s'agit là d'un problème moral autant qu'économique. $»^{2}$ Le protectionnisme des pays industriels à l'égard des plus pauvres de la planète est d'autant plus paradoxal qu'il affecte davantage les exportations de produits agricoles des PMA. Dans le cadre de la Convention de Lomé par exemple, en dépit du «libre accès accordé aux produits industriels » que la plupart des pays ACP (Afrique - Caraibes - Pacifique) n'exportent guère, les exportations de ces derniers vers l'Union européenne se heurtent - pour certains produits agricoles transformés - à des prélèvements variables augmentant en fonction du degré de transformation locale;

- l'usage croissant des mesures antidumping dans les domaines où les PVD ont réussi à pénétrer les marchés ;

- l'asymétrie entre l'état avancé de la libéralisation du commerce des biens et des services d'intérêt pour les pays développés d'une part, et les barrières au commerce des services de main-d'œuvre, typiquement fournis par les PVD, d'autre part: les marchés de la main-d'œuvre sont demeurés protégés dans les pays industriels tandis que ceux des capitaux ont été libéralisés dans les PVD. En outre, la main-d'œuvre qualifiée est devenue plus mobile alors que la main-d'œuvre non qualifiée continue de se heurter à des contraintes multiples. Il va sans dire que si cette tendance persiste, les PVD ne seront pas en mesure de développer leur compétitivité dans les secteurs qui recèlent les vrais gisements de croissance fondés sur le savoir; 
- la couverture et la sécurité d'accès peu adéquates pour les PVD dans les programmes Système généralisé de préférences (SGP) des pays industriels.

Il y a lieu d'envisager un traitement sérieux de ces déséquilibres dans le système commercial multilatéral, en insistant notamment sur la mise en œuvre complète des résultats des Cycles d'Uruguay et de Tokyo, communément dénommés unfinished business («ordre du jour non épuisé»). Il n'est au demeurant guère surprenant que le non-traitement de cet «ordre du jour non épuisé » ait accentué l'accumulation des déficits de la balance des paiements dans les PVD. Les déficits sont justement l'une des sources d'instabilité ayant conduit à la répétition de crises financières. N'est-ce pas là un exemple d'incohérence des politiques qui a privé bon nombre de PVD des dividendes de l'intégration dans le système commercial multilatéral? Il n'est guère étonnant non plus de constater que l'«ordre du jour non épuisé» porte sur les questions à même d'améliorer la balance commerciale des PVD: les textiles et l'habillement, l'agriculture, les crêtes et la progressivité tarifaires dans des domaines comme l'industrie alimentaire, les fruits et légumes, les cuirs, les chaussures, etc.

Ces tendances sont susceptibles de renforcer l'incohérence systémique dans l'édification des règles du jeu du commerce mondial. Les PVD sont confrontés à une situation asymétrique évidente: les caractéristiques du sous-développement, notamment les difficultés d'accès aux finances et à la technologie ainsi que la faible capacité administrative, inhibent leur aptitude à tirer profit des opportunités offertes par le système commercial multilatéral. C'est pourquoi les PVD avaient insisté, durant la phase préparatoire de la Conférence de Seattle, sur la nécessité d'introduire dans le projet de déclaration un constat relatif à la problématique du développement, qui devra être intégrée dans l'ordre du jour des futures négociations. Il s'agissait de poser un diagnostic de l'ensemble des problèmes auxquels les PVD sont confrontés et auxquels les négociations envisagées devraient tenter de remédier dans une perspective bien comprise de développement. A défaut de cela, toute négociation éventuelle serait fondée sur une prémisse inadéquate selon laquelle la libéralisation du commerce mondial ainsi que la codification sans cesse croissante des disciplines commerciales multilatérales englobant des nouveaux domaines représentent une fin en soi, plutôt qu'un moyen d'accélérer le développement des PVD.

\section{PROMOUVOIR LA COHÉRENCE DES POLITIQUES: UN «AGENDA POSITIF POUR LE SUD "}

L'histoire a montré qu'il ne sert à rien d'octroyer aux pays les plus démunis des préférences commerciales si elles ne s'accompagnent pas de mesures concrètes destinées à renforcer leur capacité d'offre de produits à l'exportation. Ces mesures concrètes passent par une approche fondée sur ce que le président Roosevelt a appelé l' "expérimentation audacieuse», pour éliminer le fardeau de la dette et accroître l'aide publique au développement en faveur des PVD les plus pauvres. L'absence de telles mesures pendant ces vingt-cinq dernières années a eu des effets négatifs sur le potentiel de développement des PMA, et ceux d'Afrique en particulier, qui ont vu leur part du marché mondial des matières premières décliner régulièrement de $8,6 \%$ à $3 \%$. Durent cette même période, les 68 pays ACP ont subi une baisse similaire, de 8,4\% à 3,1\%, tandis que les 48 PMA ont perdu les trois quarts de leur part de marché pour atteindre 
$1,2 \%$. Comme l'a indiqué le secrétaire général de la $\mathrm{CNUCED}^{3}$, ce phénomène prend des proportions quasi dramatiques dès lors qu'on sait que les exportations africaines de matières premières (excluant l'Afrique du Sud) représentaient quatre fois la valeur de celles du Brésil au début des années 1970; de nos jours, la part de l'Afrique est inférieure à celle du Brésil: 22,2 milliards de dollars pour l'Afrique en 1997 contre 23,3 milliards pour le Brésil. L'incohérence de cet état de choses réside dans le fait que les matières premières constituent près de trois quarts des exportations totales de l'Afrique. Le paradoxe révélé par ces données est encore plus frappant lorsque l'on constate que pendant ces vingt dernières années, la part des pays industriels dans les exportations globales de matières premières a sensiblement augmenté. Ces tendances soulèvent un problème de cohérence éthique: le paradigme de croissance des années 1990 a accentué le fossé Nord-Sud, cependant que la marginalisation des pays les plus faibles de la communauté des nations semble aller de pair avec le renforcement des disciplines commerciales multilatérales et la réduction sensible des flux financiers publics vers le Sud.

A la lumière de ces tendances se pose la question suivante: que faire pour intégrer le volet du développement dans les perspectives inhérentes aux enjeux futurs d'éventuelles négociations après Seattle, afin d'équilibrer le système commercial multilatéral ? En d'autres termes, comment remédier à l'absence de cohérence des politiques dans le domaine du commerce? On peut proposer plusieurs réponses à cette question. Il s’agit de substituer au slogan Trade not aid («Le commerce plutôt que l'aide ») celui de l'expansion des exportations grâce à l'accès au marché plutôt que le recours par les PVD aux capitaux volatiles destinés à compenser la faiblesse des revenus de l'exportation. Une autre réponse consiste à œuvrer dans deux directions: plus d'accès pour les PVD aux nouveaux investissements directs générateurs de capacités additionnelles à l'exportation et de main-d'œuvre qualifiée, et plus de flexibilité accordée aux PVD, notamment les plus pauvres, afin qu'ils utilisent la panoplie d'instruments de politiques économiques pour promouvoir le processus complexe du développement.

La cohérence des politiques dans le domaine du commerce exige que l'on résiste à la tentation d'utiliser l'élaboration de règles commerciales multilatérales comme instrument de gouvernance globale qui prive les PVD des politiques actives pour acquérir l'avantage compétitif. Cette démarche est d'autant plus justifiée que les pays industrialisés ont amplement fait usage de telles politiques économiques actives - comme les subventions - dans leur processus historique de développement.

La dimension éthique de la cohérence des politiques revêt une pertinence plus significative dès lors que l'on appréhende les déséquilibres systémiques inhérents aux relations commerciales Nord-Sud. Etant donné que les marchés présentent d'immenses différences en taille et en pouvoir d'achat, comment peut-on palier le fait que l'inévitable asymétrie du «pouvoir du marché» aura tendance à aggraver les inégalités excessives Nord-Sud?

La réponse à cette question réside dans une redéfinition de la concurrence dans une perspective de développement, en mettant un accent particulier sur un réexamen du Traitement spécial et différencié. Une telle démarche, fondée sur une

3. R. Ricupero, «Capacity and Institution Building in Developing Countries", communication au Geneva Week, 1.11 .99 , p. 7. 
dose de sélectivité, aurait pour objectif de permettre aux PVD d'affronter la dynamique de changements dans l'économie mondiale découlant des évolutions technologiques rapides. Le réexamen du Traitement spécial et différencié devrait privilégier les PVD plus vulnérables, notamment les pays les moins avancés, et comporter en particulier deux volets: des améliorations dans les tarifs préférentiels, mais aussi dans les périodes transitoires avant l'application des règles. Le renforcement des capacités d'offre de produits et de services pour les pays les plus faibles, notamment à travers l'accès à l'information et à la connaissance, leur permettra de jouir du «droit de participer aux échanges mondiaux».

Les plaintes des PVD dans le domaine commercial sont connues et sont de trois ordres: les résultats du Cycle d'Uruguay et leur mise en œuvre ont peu contribué à l'amélioration de l'accès au marché pour les exportations des PVD; en outre, la plupart des PVD considèrent que certaines règles se sont avérées à leur détriment dans plusieurs domaines d'importance pour le développement, notamment les droits de propriété intellectuelle et l'utilisation des subventions, tandis que le Traitement spécial et différencié prévu pour eux dans les accords n'a pas été adéquat; enfin, des capacités humaines et institutionnelles faibles et le manque de financement n'ont pas permis aux PVD d' «activer» leurs avantages commerciaux et d'user du mécanisme de règlement des différends. Le fait que seuls $2 \%$ des activités de coopération technique portent sur le commerce constitue une des incohérences des politiques à cet égard.

Que faire pour remédier à ces déficits dans les perspectives de développement? Il y a lieu d'aider les PVD à aborder les futures négociations commerciales avec ce que la CNUCED a appelé un "Agenda positif pour le Sud» ${ }^{4}$. Cette plateforme - conçue par la CNUCED - a pour objectif de permettre aux PVD de tirer les leçons du Cycle d'Uruguay pour élaborer des propositions ${ }^{5}$ techniques détaillées sur les sujets pertinents, dans le cadre du processus préparatoire pour la Conférence de Seattle, avec l'appui technique et analytique de la CNUCED. Suite en grande partie à cette approche, à la date du 10 novembre 1999 , sur les 240 propositions concernant 20 thèmes soumises à l'OMC dans ce processus préparatoire, plus de $50 \%$ émanent des PVD.

L' "Agenda positif» aura pour impact éventuel d'introduire plus de cohérence dans le système commercial, et ce dans plusieurs directions:

๖ l'accès au marché: le démantèlement de l'arsenal de protection tarifaire - notamment les crêtes tarifaires variant de $12 \%$ à $300 \%$ - affectant les produits agricoles et industriels d'intérêt à l'exportation pour les PVD, ainsi que le libre accès pour toutes les importations émanant des PMA dans les pays industriels;

- l'antidumping: l'usage abusif de ces mesures et d'autres formes de contingentement est perçu par les PVD comme un facteur amoindrissant les avantages qu'ils pourraient tirer de la libéralisation du commerce;

4. UNCTAD Secretariat, A Positive Agenda for Developing Countries in Future Trade Negotiations, 1999 (disponible sur le site Internet de la CNUCED, p. www.unctad.org/en/docs/soposag.pdf).

5. Durant le Cycle d'Uruguay, exception faite d'une dizaine de pays d'Asie et d'Amérique latine, la grande majorité des PVD savaient ce qu'ils ne souhaitaient pas voir incorporé dans les accords, mais non ce qu'ils voulaient y inclure.

6. Certaines de ces propositions ont été au demeurant soumises de façon conjointe avec des pays développés. 
- la réforme de l'agriculture: les obstacles auxquels se heurtent les PVD sont connus et ont pour noms les soutiens aux producteurs, les subventions à l'exportation dans les pays industriels et les difficultés rencontrées dans la mise en œuvre du système de quotas;

- les services: la flexibilité de l'architecture de l'Accord général sur le commerce des services doit être préservée, notamment par des engagements spécifiques négociés et par l'accès aux réseaux d'information et de distribution et aux technologies;

- les médicaments essentiels : notamment la reconnaissance du droit des PVD d'accéder à ces médicaments à prix raisonnables, conformément à l'article 31 de l'Accord sur les aspects des droits de propriété intellectuelle liés au commerce (ADPIC).

Bien d'autres domaines sont couverts par l'«Agenda positif», dont l'inclusion dans les accords envisagés permettra d'accroître sensiblement la cohérence du système commercial multilatéral, ce dans une perspective de développement.

\section{CONCLUSION}

Un traitement sérieux de la problématique de la cohérence des politiques dans les relations Nord-Sud devrait être fondé sur les éléments suivants: une reconnaissance complète de la nécessité de «niveler le terrain de jeu pour tous » dans le système commercial multilatéral, l'élaboration d'une approche multidimensionnelle en matière de stratégie de développement, la promotion de l'équité dans les systèmes, structures et arrangements qui sous-tendent les relations économiques internationales - les rapports Nord-Sud en particulier -, et enfin une revitalisation authentique du Traitement spécial et différencié, notamment en faveur des pays les plus vulnérables. La matérialisation des dividendes de l'intégration dans l'économie mondialisée ne deviendra réalité - pour les PVD - qu'à la faveur d'un renforcement de la cohérence des politiques en matière de coopération internationale au développement. Une adaptation des architectures financière, commerciale et de concurrence, dans une perspective de développement, devrait présider à une revitalisation de la cohérence des politiques à l'aube du prochain millénaire.

La cohérence des politiques dans les relations Nord-Sud exige l'élaboration d'un mécanisme de régulation de la mondialisation. Face aux risques d'émergence d'une «société de marché», la communauté internationale dans ses composantes multiformes - y compris la société civile - devrait promouvoir une globalisation plus équitable. Afin que le processus d'unification des marchés jouisse de l'adhésion de l'opinion publique mondiale, il devra inclure plutôt qu'exclure les pays les plus pauvres et les plus vulnérables. Il est d'autant plus impératif de promouvoir une plus grande cohérence des politiques que la combinaison de la pauvreté et des flux d'information sans cesse croissants peut se révéler un mélange explosif à bien des égards. L'espoir réside dans la promotion d'une deuxième mondialisation, celle de la civilisation, de la culture, de la citoyenneté, celle de l'homme tout court. 